\title{
Postoperative trans women in sexual health clinics: managing common problems after vaginoplasty
}

\author{
Tara Suchak, ${ }^{1}$ Jane Hussey, ${ }^{2}$ Manjit Takhar, ${ }^{3}$ James Bellringer ${ }^{4}$
}

\begin{abstract}
'Post-CCT Fellow, Sexual Health, City Hospitals Sunderland NHS Foundation Trust, Sunderland, UK

${ }^{2}$ Consultant, Genitourinary Medicine, City Hospitals Sunderland NHS Foundation Trust, Sunderland, UK

${ }^{3}$ Clinical Nurse Specialist, Imperial College Healthcare NHS Trust, London, UK

${ }^{4}$ Gender Reassignment Surgeon, Parkside Hospital, London, UK
\end{abstract}

\section{Correspondence to} Dr Jane Hussey, Genitourinary Medicine Department, City Hospitals Sunderland, Kayll Road, Sunderland SR4 7TP, UK; Jane.Hussey@chsft.nhs.uk

Received 10 September 2014 Revised 25 February 2015 Accepted 28 April 2015 Published Online First 10 June 2015

CrossMark

To cite: Suchak T, Hussey J, Takhar M, et al. J Fam Plann Reprod Health Care 2015;41:245-247.

\section{BACKGROUND}

UK figures estimate that in 1998 there were 3170 people over the age of 15 years assigned as male at birth who had presented with gender dysphoria. This figure is comparable to that found in the Netherlands where 2440 have presented; ${ }^{1}$ however, far fewer people actually undergo sex reassignment surgery. Recent statistics from the Netherlands indicate that about 1 in 12000 natal males undergo sex-reassignment and about 1 in 34000 natal females. ${ }^{2}$ Since April 2013, English gender identity services have been among the specialised services commissioned centrally by NHS England and this body is therefore responsible for commissioning transgender surgical services.

The growth in the incidence of revealed gender dysphoria amongst both young and adult people has major implications for commissioners and providers of public services. The present annual requirement is 480 genital and gonadal male-to-female reassignment procedures. There are currently three units in the UK offering this surgery for National Health Service (NHS) patients. Prior to surgery trans women will have had extensive evaluation, including blood tests, advice on smoking, alcohol and obesity, and psychological/psychiatric evaluation. They usually begin to take female hormones after 3 months of transition, aiming to encourage development of breast buds and alter muscle and fat distribution. Some patients may elect at this stage to have breast surgery. Before genital surgery can be considered the patient must have demonstrated they have lived for 1 year full-time as a woman. Figure 1 shows a typical post-surgical result.

A trans person who has lived exclusively in their identified gender for at least 2 years (as required by the Gender
Recognition Act 2004) can apply for a gender recognition certificate (GRC). This is independent of whether gender reassignment surgery has taken place. Once a trans person has a GRC they can then obtain a new birth certificate. ${ }^{3}$ The trans person will also have new hospital records in a new name. It is good practice for health providers to take practical steps to ensure that gender reassignment is not casually visible in records or communicated without the informed consent of the user. Consent must always be sought (and documented) for all medical correspondence where the surgery or life before surgery when living as a different gender is mentioned (exceptions include an order of court and prevention or investigation of crime). ${ }^{4}{ }^{5}$ It is advisable to seek medico-legal advice before disclosing.

Not all trans women opt to undergo vaginoplasty. Patients have free choice as to how much surgery they wish to undertake. Trans women often live a considerable distance from where their surgery was performed and as a result many elect to see their own general practitioner or local Sexual Health Clinic if they have postoperative problems. Fortunately reported complications following surgery are rare. Lawrence summarised 15 papers investigating 232 cases of vaginoplasty surgery; 13 reported rectal-vaginal fistula, 39 reported vaginal stenosis and 33 urethral stenosis; ${ }^{6}$ however, it is likely that there is significant under-reporting of complications.

Here we present some examples of post-vaginoplasty problems presenting to a Sexual Health Service in the North East of England, and how they were managed.

\section{CASE 1: VAGINAL HAIR}

Six months following surgery a trans woman is experiencing painful neovaginal dilatation, dyspareunia and an intermittent 


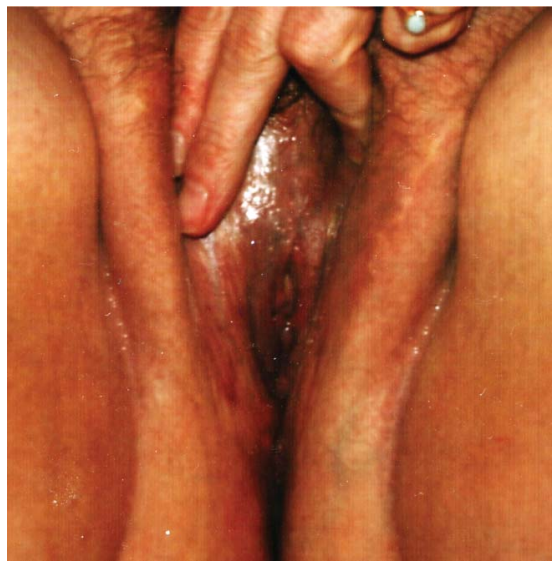

Figure 1 Photograph illustrating the typical results of male-to-female reassignment genital surgery.

foul smelling discharge, worse after dilating. She had had laser therapy to her scrotum prior to surgery. On examination her perineum has long hairs present which enter the introitus and speculum examination reveals multiple internal hairs with thick discharge present amongst them.

The neovagina is usually created by forming a cavity in the perineum and pelvis, which is then lined with penile skin, scrotal skin, or a combination of both. Dilatation is required postoperatively to ensure the neovagina maintains its depth and girth in patients not having regular penetrative intercourse. To prevent intravaginal hair growth following the use of hairbearing grafts (those using scrotal skin), patients undergoing vaginoplasty should have electrolysis prior to surgery. ${ }^{7}$ In late follow-up of 70 postoperative trans women [mean 3 years (range 9 months to 8 years)], $29 \%$ were concerned about vaginal hair growth. ${ }^{8}$

Should internal hair be present postoperatively, options include plucking (using a speculum and

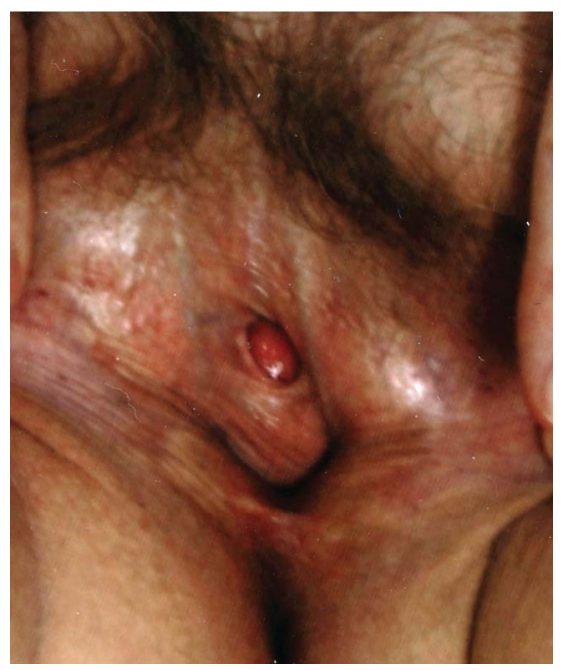

Figure 2 Example of a urethral meatus pointing forwards, with a bulky corpus spongiosum remnant. removing by pulling with sponge forceps) or the use of hair removal creams (such as Veet ${ }^{\circledR}$ ). ${ }^{7}$ These should be patch-tested first, then inserted internally, left for the allotted time and then removed by douching thoroughly. Hair regrowth to the perineum can be removed by repeated electrolysis.

This trans woman was referred locally for further electrolysis externally and recommended to douche regularly to avoid retained hair accumulation and secondary inflammation.

\section{CASE 2: THE SHORT VAGINA}

Five years following surgery a trans woman attends complaining of her vagina being too short and sex uncomfortable - she is unable to fully insert her dilators and has measured a depth of $6 \mathrm{~cm}$ herself. On examination her vulva has a good cosmetic postoperative result. Internal inspection shows a short vagina, although girth appears normal.

Although studies have shown that the majority of trans women are happy with the length of their vagina post-surgery, $12 \%$ have been reported to have inadequate vaginal length. ${ }^{8} 9$ To increase depth in a neovagina further surgery is required, most commonly using an isolated bowel segment on its vascular pedicle. Otherwise altering sexual practice may be needed to take into account the maximum size of a neovagina. ${ }^{7}$

This trans woman opted to alter sexual practice and not to undertake further surgery.

\section{CASE 3: URINATION DIFFICULTY}

Two years following surgery a trans woman attends complaining of urine spraying upwards over her abdomen. Her clothes are getting wet and she now feels unable to leave the house. On examination her urethral meatus is not flat to her vulva and introitus but opens anteriorly.

Figure 2 shows an example of a urethral meatus pointing forwards, with a bulky corpus spongiosum remnant. Urination problems are common postsurgery with up to $20 \%$ of postoperative trans women requiring minor corrective urethral surgery. ${ }^{8} \quad 10$ Surgery in the form of urethral revision is the only solution to correct this. This will remove much of the residual erectile tissue of the corpus spongiosum and reposition the urethra with a more ventral opening. ${ }^{9}$

This trans woman was referred for further surgery.

\section{CASE 4: VAGINAL DISCHARGE}

Nine months following surgery a trans woman attends complaining of intermittent blood-stained discharge, worse after dilating. Speculum examination shows an area of red granulation tissue in the vault of the neovagina.

The location of this area of granulation makes it especially difficult to treat and it may be related to the presence of infection. Silver nitrate sticks are used to 
reduce fibroblast production and are one treatment for over-granulation, with good results in practice. ${ }^{11}$

This trans woman received fortnightly application of silver nitrate and required a couple of courses of metronidazole to resolve her symptoms and signs.

\section{DISCUSSION}

With only three NHS units in the country offering gender reassignment surgery, trans women have to travel long distances for minor postoperative problems and concerns. Transgender issues are not currently on the specialist genitourinary medicine training curriculum in the UK, other than in the context of managing HIV in transgender patients (whose risk of HIV may indeed be very high), ${ }^{12}$ and they are also not on the Community Sexual and Reproductive Health curriculum. We feel that this needs addressing, as demonstrated by the cases presented. Many health care providers are unaware of the Gender Recognition Act 2004 and the need to maintain confidentiality regarding gender reassignment surgery even if relevant to the referral, unless explicit consent has been given. This needs highlighting to those working in sexual and reproductive healthcare (SRH).

Sexual health specialists are well placed to provide holistic sexual health care to transgender patients. Offering a local service for trans women to access through SRH clinics for assessment of these problems is more convenient for them and helps relieve pressure on specialist centres. Sexually transmitted infection screening and health promotion can also be addressed. This would also reduce patient transport costs. Advice can be sought if needed from the patient's surgeon. Interested SRH services should contact their local gender reassignment service to establish local care pathways.

Competing interests None declared.
Provenance and peer review Not commissioned; externally peer reviewed.

Patient consent Obtained.

\section{REFERENCES}

1 Reed B, Rhodes S, Schofield P, et al. Gender Variance in the UK: Prevalence, Incidence, Growth and Geographic Distribution. Gender Identity Research and Education Society, 2009. http://www.gires.org.uk [accessed 14 May 2014].

2 Transgender Issues: A Fact Sheet. http://www.transgenderlaw. org/resources/transfactsheet.pdf [accessed 2 November 2014].

3 Frequently Asked Questions Guidance for Gender Recognition Panel. http://www.justice.gov.uk/tribunals/gender-recognitionpanel/faqs [accessed 14 May 2014].

4 Gender Recognition Act 2004. http://www.Legislation.gov.uk/ ukpga/2004/7/contents [accessed 14 May 2014].

5 Trans: A Practical Guide for the NHS. London, UK: Department of Health, 2008.

6 Lawrence A. Patient-reported complications and functional outcomes of male to female sex reassignment surgery. Arch Sex Behav 2006;35:717-727.

7 Bowman C, Goldberg S. Care of the Patient Undergoing Sex Reassignment Surgery. Vancouver, Canada: Vancouver Coastal Health, Transcend Transgender Support \& Education Society, and the Canadian Rainbow Health Coalition, 2006.

8 Goddard JC, Vickery RM, Qureshi A, et al. Feminizing genitoplasty in adult transsexuals: early and long term surgical results. BJU Int 2007;100:607-613.

9 Krege S, Bex A, Lummen G, et al. Male to female transsexualism: a technique, results and long term follow up in 66 patients. BJU Int 2001;88:396-402.

10 Hoebeke P, Selvaggi G, Ceulemans P, et al. Impact of sex reassignment surgery on lower urinary tract function. Eur Urol 2005;47:398-402.

11 Borkowski L. G tube care: managing hypergranulation tissue. Nursing 2005;35:24.

12 Baral S, Poteat T, Stromdahl S, et al. Worldwide burden of HIV in transgender women: a systematic review and meta-analysis. Lancet Infect Dis 2013;13:214-222. 